Journal of Mathematics and Statistics 7 (3): 169-176, 2011

ISSN 1549-3644

(C) 2011 Science Publications

\title{
Reliability Equivalence Factors of a System with 2 Non-identical Mixed Lifetimes and Delayed Time
}

\author{
${ }^{1}$ Abdelfattah Mustafa and ${ }^{2}$ Adel A. El-Faheem \\ ${ }^{1}$ Department of Mathematics, Faculty of Science, \\ Mansoura University, Mansoura 35516, Egypt \\ ${ }^{2}$ Department of Mathematics, Faculty of Science, \\ South Valley University, Aswan, Egypt
}

\begin{abstract}
Problem statement: In reliability theory and operations research, there are many methods and techniques to improve the performance of a system. The aim of this study is to generalize reliability equivalence technique to apply it to a mixed failure lifetimes system $f_{1}(t), f_{2}(t)$ with delayed time. Approach: We shall improve the system by using some reliability techniques: (i) reducing the failure for some lifetimes; (ii) add hot duplication components; (iii) add cold duplication components; (iv) add cold duplication components with imperfect switches. We start by establishing two different types of reliability equivalence factors, the Survival Reliability Equivalence (SRE) and Mean Reliability Equivalence (MRE) factors. Also, we introduced some numerical results. Results: The system reliability function and mean time to failure will be used as reference of the system performances. For this reason, we obtain the reliability functions and mean time to failures of the original and improved systems using each improving methods. Conclusion: The results can be used to distinguish between the original and improved systems performances and calculate the equivalent between different cases of improving methods.
\end{abstract}

Key words: Mixture distributions, reliability equivalence, improving system, exponential distribution, improving methods, systems performances, improving methods, equivalence factors, operations research, reduction method, duplication method

\section{INTRODUCTION}

Operations Research, in its various fields, is concerned with the problem of system performance in the best possible way. In reliability theory, one way to improve the performance of a system is to use the redundancy method. There are two main such methods: Hot duplication method; in this case, it is assumed that some of the system components are duplicated in parallel. Cold duplication method: In this case, it is assumed that some of the system components are duplicated in parallel via a perfect switch. Unfortunately, for many different reasons, such as space limitation, high cost, it is not always possible to improve a system by duplicating some or all of its components, Rushdi and Alsulami (2007) and Haggag (2009). For example, satellites and space aircrafts have limited space which doesn't allow component duplication. Also, some microchips are so expansive that manufacturers cannot afford to duplicate them. In such cases where duplication is not possible, the engineer turns to another well-known method in reliability theory, the so-called reduction method, Mokaddis et al. (2009) and Gupta et al. (2006). In this method, it is assumed that the failure rates of some of the system components are reduced by a factor $\rho$, $0<\rho<1$. Now, once the reduction method is adopted, the main problem facing the engineer is to decide to what degree the failure rate should be decreased in order to improve the system. To solve this problem, one can make equivalence between the reduction method and the duplication method based on some reliability measures. In other words, the design of the system improved by the reduction method should be equivalent to the design of the system improved by one of the duplication methods. The comparison of the designs produces the so-called reliability equivalence factors, Sarhan et al. (2000). Sarhan and Mustafa (2006)

Corresponding Author: Abdelfatah Mustafa, Department of Mathematics, Faculty of Science, Mansoura University, Mansoura 35516, Egypt 


\section{J. Math. \& Stat., 7 (3): 169-176, 2011}

introduced different vectors of the reliability equivalence factors of a series system consists of $\mathrm{n}$ independent and non- identical components. Mustafa, et al. (2007), introduced three different types of the reliability equivalence factors of a parallel-series system consists of four independent and identical components. The lifetime of the system component is assumed to follow exponential distribution. Xia and Zhang (2007), applied the concept of the reliability equivalence on $n$ components parallel system with non-constant failure rates, authors considered the life distribution of the components are Gamma distribution. Gamma distribution can be reduced to the exponential distribution when $\mathrm{n}=1$, this means $\operatorname{GD}(1, \lambda) \equiv \operatorname{ED}(\lambda)$ Mustafa (2009b) applied the concept of reliability equivalence when the components have mixture Weibull failure rates, Mustafa (2008), generalized reliability equivalence techniques to apply to a system consists of two independent and non-identical components connected in series (parallel) system, that have constant failure rates. The system improved by using one component only with redundant methods. Sarhan (2002), introduced equivalence factors of a general series-parallel system, Sarhan, et al. (2008), introduced equivalence factors of a general parallelseries system and assumed that all components are independent and follow the exponential distribution with the same parameter, $\lambda>0$. Mustafa (2009a) studied the reliability equivalence factor of a series system. The failure rates of the system components are functions of time $\mathrm{t}$ and introduced two cases of non-constant failure rates (i) Weibull distribution (ii) linear increasing failure rate distribution. There are two methods are used to improve the given system. Mustafa (2009b) introduced reliability equivalence factors for some systems with mixture weibull failure rates and studied two cases (i) the mixture of two stages of life time distribution with weibull failure rates, (ii) the mixture of two stages failure rates with weibull distribution. Mustafa and El-Bassiouny (2009), introduced the reliability equivalence factor of the system such that the failure rates of the system's components are functions of time t, studied two cases (i) the life time distribution of a components has two stages with increasing failure rates, (ii) the failure rates of the components have the two stages. Mustafa et al. (2009), applied the reliability equivalence techniques to a system consists of $n$ independent and non-identical components connected in series system, which have mixing constant failure rates. Mustafa and El-Faheem (2011), generalized reliability equivalence technique to apply it to a system consists of $\mathrm{m}$ independent and non-identical lifetimes distributions, with mixed failure lifetimes $\mathrm{f}_{1}(\mathrm{t})$, $\mathrm{f}_{2}(\mathrm{t}), \ldots, \mathrm{f}_{\mathrm{m}}(\mathrm{t})$. In this article, we consider a system with mixing lifetimes, delayed time. This modal is applicable when each component or product experience two (or more) failure modes. For example, a mechanical component, such as a load-carrying bearing or a cutting tool, may fail due to wear-out or when the applied stress exceeds the design strength of component material. Since the component or the tool can fail in either of the failure modes, it is then appropriate to describe the hazard rate by a mixed model, it is expressed as follows, Everitt and Hand (1981) and Teamah and El-Bar (2009):

$f(t)=p_{1} f_{1}(t)+p_{2} f_{2}(t)$

where, $0 \leq \mathrm{p}_{\mathrm{i}} \leq 1, \mathrm{i}=1,2, \mathrm{p}_{1}+\mathrm{p}_{2}=1$, the quantity $\mathrm{p}_{1}$ is the probability that the component or the tool fails in the first failure mode and $\mathrm{p}_{2}$ is the probability that it fails in the second failure, if the second failure mode occurs after a delay time $\delta$ from the first failure mode, then:

$f_{d}(t)=p_{1} f_{1}(t)+p_{2} f_{2}(t-\delta), 0 \leq \delta \leq t$

Original system: We derive the reliability function and mean time to failure for a system with two nonidentical mixing lifetime distribution and delayed time. Assuming the failure rates of the two types of mixed lifetimes are, $\lambda_{i} i=1,2$, that is the failure time for the system is given as, El-Said and El-Sherbeny (2005):

$\mathrm{f}(\mathrm{t})=\lambda_{1} \mathrm{p}_{1} \exp \left\{-\lambda_{1} \mathrm{t}\right\}+\lambda_{2} \mathrm{p}_{2} \exp \left\{-\lambda_{2}(\mathrm{t}-\delta)\right\}$

Reliability function $R(t)$, for the original system can be obtained as follows:

$\mathrm{R}(\mathrm{t})=\mathrm{p}_{1} \exp \left\{-\lambda_{1} \mathrm{t}\right\}+\mathrm{p}_{2} \exp \left\{-\lambda_{2}(\mathrm{t}-\delta)\right\}$

From Eq. 2b, one can easily obtain the mean time to failure, say MTTF, as follows:

MTTF $=\frac{p_{1}}{\lambda_{1}}+\frac{p_{2} \exp \left\{\lambda_{2} \delta\right\}}{\lambda_{2}}$

The improved systems: The quality of the system reliability can be improved by using four different methods of the system improvements.

Reduction method: Let $\mathrm{R}_{\rho}(\mathrm{t})$ denote to the reliability function of the improved system, when the failure rate of the mixing components are reduced by the factor $\rho_{i}$, 
$0<\rho_{i}<1, i=1,2$. One can obtain the function $R_{\rho}(t)$, as follows:

$\mathrm{R}_{\rho}(\mathrm{t})=\mathrm{p}_{1} \exp \left\{-\rho_{1} \lambda_{1} \mathrm{t}\right\}+\mathrm{p}_{2} \exp \left\{-\rho_{2} \lambda_{2}(\mathrm{t}-\delta)\right\}$

From Eq. 3a, the mean time to failure of the improved system, say $\mathrm{MTTF}_{\rho}$, becomes:

$$
\operatorname{MTTF}_{\rho}=\frac{p_{1}}{\lambda_{1} \rho_{1}}+\frac{p_{2} \exp \left\{\rho_{2} \lambda_{2} \delta\right\}}{\rho_{2} \lambda_{2}}
$$

Hot duplication method: Let $\mathrm{R}^{\mathrm{H}}$ (t) be the reliability function of the improved system obtained by assuming hot duplications of the system component. The function $R^{H}(t)$ can be obtained as follows, Lewis (1996):

$R^{H}(t)=[2-R(t)] R(t)$

Where:

$$
\mathrm{R}(\mathrm{t})=\mathrm{p}_{1} \exp \left\{-\lambda_{1} \mathrm{t}\right\}+\mathrm{p}_{2} \exp \left\{-\lambda_{2}(\mathrm{t}-\delta)\right\}
$$

Let $\mathrm{MTTF}^{\mathrm{H}}$ be the mean time to failure of the improved system assuming hot duplication method. Using Eq. 3c, one can deduce MTTF ${ }^{\mathrm{H}}$ as:

$$
\begin{aligned}
& \operatorname{MTTF}^{\mathrm{H}}=\frac{\left(4-\mathrm{p}_{1}\right) \mathrm{p}_{1}}{2 \lambda_{1}}+\frac{\mathrm{p}_{2} \exp \left\{\lambda_{2} \delta\right\}}{2 \lambda_{2}} \\
& {\left[4-\mathrm{p}_{2} \exp \left\{\lambda_{2} \delta\right\}\right]-\frac{2 \mathrm{p}_{1} \mathrm{p}_{2} \exp \left\{\lambda_{2} \delta\right\}}{\lambda_{1}+\lambda_{2}}} \\
& =\mathrm{MTTF}+\frac{\left(2-\mathrm{p}_{1}\right) \mathrm{p}_{1}}{2 \lambda_{1}}+\frac{\mathrm{p}_{2} \exp \left\{\lambda_{2} \delta\right\}}{2 \lambda_{2}} \\
& {\left[2-\mathrm{p}_{2} \exp \left\{\lambda_{2} \delta\right\}\right]-\frac{2 \mathrm{p}_{1} \mathrm{p}_{2} \exp \left\{\lambda_{2} \delta\right\}}{\lambda_{1}+\lambda_{2}}}
\end{aligned}
$$

That is, hot duplication of a single component increases the mean time to system failure by the amount:

$$
\frac{\left(2-\mathrm{p}_{1}\right) \mathrm{p}_{1}}{2 \lambda_{1}}+\frac{\mathrm{p}_{2} \exp \left\{\lambda_{2} \delta\right\}}{2 \lambda_{2}}\left[2-\mathrm{p}_{2} \exp \left\{\lambda_{2} \delta\right\}\right]-\frac{2 \mathrm{p}_{1} \mathrm{p}_{2} \exp \left\{\lambda_{2} \delta\right\}}{\lambda_{1}+\lambda_{2}}
$$

Cold duplication method: Let $\mathrm{R}^{\mathrm{C}}(\mathrm{t})$ be the reliability function of the improved system obtained by assuming cold duplications of the mixing system components. The function $\mathrm{R}^{\mathrm{C}}(\mathrm{t})$ can be obtained as follows, Billinton and Allan (1983):

$$
\begin{aligned}
& \mathrm{R}^{\mathrm{C}}(\mathrm{t})=\mathrm{p}_{1}^{2}\left(1+\lambda_{1} \mathrm{t}\right) \exp \left\{-\lambda_{1} \mathrm{t}\right\}+\frac{2 \mathrm{p}_{1} \mathrm{p}_{2} \exp \left\{\lambda_{2} \delta\right\}}{\lambda_{2}-\lambda_{1}} \\
& {\left[\begin{array}{l}
\lambda_{2} \exp \left\{-\lambda_{1} \mathrm{t}\right\} \\
-\lambda_{1} \exp \left\{-\lambda_{2} \mathrm{t}\right\}
\end{array}\right]+\mathrm{p}_{2}^{2}\left(1+\lambda_{2} \mathrm{t}\right) \exp \left\{-\lambda_{2}(\mathrm{t}-2 \delta)\right\}}
\end{aligned}
$$

From Eq. 3e, the mean time to failure of the improved system, say $\mathrm{MTTF}^{\mathrm{C}}$, assuming cold duplications method is given as:

$$
\begin{gathered}
\operatorname{MTTF}^{\mathrm{C}}=2\left[\frac{\mathrm{p}_{1}^{2}}{\lambda_{1}}+\frac{\mathrm{p}_{1} \mathrm{p}_{2}\left(\lambda_{1}+\lambda_{2}\right) \exp \left\{\lambda_{2} \delta\right\}}{\lambda_{1} \lambda_{2}}+\frac{\mathrm{p}_{2}^{2} \exp \left\{2 \delta \lambda_{2}\right\}}{\lambda_{2}}\right] \\
=\mathrm{MTTF}+\frac{\mathrm{p}_{1}\left(2 \mathrm{p}_{1}-1\right)}{\lambda_{1}}+\frac{\mathrm{p}_{2}\left[2 \mathrm{p}_{1}\left(\lambda_{1}+\lambda_{2}\right)-\lambda_{1}\right] \exp \left\{\lambda_{2} \delta\right\}}{\lambda_{1} \lambda_{2}} \\
+\frac{2 \mathrm{p}_{2}^{2} \exp \left\{2 \delta \lambda_{2}\right\}}{\lambda_{2}}
\end{gathered}
$$

That is, cold duplication of the system component increases the mean time to system failure by the amount:

$$
\left[\begin{array}{c}
\frac{\mathrm{p}_{1}\left(2 \mathrm{p}_{1}-1\right)}{\lambda_{1}}+\frac{\mathrm{p}_{2}\left[2 \mathrm{p}_{1}\left(\lambda_{1}+\lambda_{2}\right)-\lambda_{1}\right] \exp \left\{\lambda_{2} \delta\right\}}{\lambda_{1} \lambda_{2}} \\
+\frac{2 \mathrm{p}_{2}^{2} \exp \left\{2 \delta \lambda_{2}\right\}}{\lambda_{2}}
\end{array}\right]
$$

Imperfect switching duplication method: Let us consider now that, the system reliability can be improved assuming cold duplication method with imperfect switch of the mixing component. In such method, it is assumed that the component is connected by a cold redundant standby component via a random switch having a constant failure rate, say $\beta$. Let $\mathrm{R}^{\mathrm{I}}(\mathrm{t})$ be the reliability function of the improved system when the system component is improved according to the cold duplication method with imperfect switch for the mixing components. The function $R^{I}(t)$ is given as follows:

$$
\begin{gathered}
\mathrm{R}^{\mathrm{I}}(\mathrm{t})=\frac{\mathrm{p}_{1}^{2}}{\beta}\left[\lambda_{1}+\beta-\lambda_{1} \exp \{-\beta \mathrm{t}\}\right] \exp \left\{-\lambda_{1} \mathrm{t}\right\} \\
+\frac{\mathrm{p}_{1} \mathrm{p}_{2}}{\lambda_{1}+\beta-\lambda_{2}}\left[\begin{array}{l}
\left(\lambda_{1}+\beta\right) \exp \left\{-\lambda_{2} \mathrm{t}\right\} \\
-\lambda_{2} \exp \left\{-\left(\beta+\lambda_{1}\right) \mathrm{t}\right\}
\end{array}\right] \\
\exp \left\{\delta \lambda_{2}\right\}+\frac{\mathrm{p}_{1} \mathrm{p}_{2}}{\lambda_{2}+\beta-\lambda_{1}}\left[\begin{array}{l}
\left(\lambda_{2}+\beta\right) \exp \left\{-\lambda_{1} \mathrm{t}\right\} \\
-\lambda_{1} \exp \left\{-\left(\beta+\lambda_{2}\right) \mathrm{t}\right\}
\end{array}\right] \\
\exp \left\{\delta \lambda_{2}\right\}+\frac{\mathrm{p}_{2}^{2}}{\beta}\left[\lambda_{2}+\beta-\lambda_{2} \exp \{-\beta \mathrm{t}\}\right] \\
\exp \left\{-\lambda_{2}(\mathrm{t}-2 \delta)\right\}
\end{gathered}
$$

From Eq. 3g, the mean time to failure of the improved system, say MTTF ${ }^{\mathrm{I}}$ is given by: 


$$
\begin{aligned}
& \operatorname{MTTF}^{\mathrm{I}}=\frac{\left(2 \lambda_{1}+\beta\right) \mathrm{p}_{1}^{2}}{\lambda_{1}\left(\lambda_{1}+\beta\right)}+ \\
& \frac{p_{1} p_{2}\left(\lambda_{1}+\beta+\lambda_{2}\right)\left[2 \lambda_{1} \lambda_{2}+\left(\lambda_{1}+\lambda_{2}\right) \beta\right] \exp \left\{\lambda_{2} \delta\right\}}{\lambda_{1} \lambda_{2}\left(\lambda_{1}+\beta\right)\left(\lambda_{2}+\beta\right)} \\
& +\frac{\mathrm{p}_{2}^{2}\left(2 \lambda_{2}+\beta\right) \exp \left\{2 \lambda_{2} \delta\right\}}{\lambda_{2}\left(\lambda_{2}+\beta\right)} \\
& =\operatorname{MTTF}+\frac{\mathrm{p}_{1}\left[\left(2 \mathrm{p}_{1}-1\right) \lambda_{1}+\left(\mathrm{p}_{1}-1\right) \beta\right]}{\lambda_{1}\left(\lambda_{1}+\beta\right)} \\
& +\frac{p_{2}^{2}\left(2 \lambda_{2}+\beta\right) \exp \left\{2 \delta \lambda_{2}\right\}}{\lambda_{2}\left(\lambda_{2}+\beta\right)} \\
& +\frac{\mathrm{p}_{2}\left\{\begin{array}{l}
\left.\left(\lambda_{1}+\lambda_{2}+\beta\right)\left[\begin{array}{l}
\mathrm{p}_{1} \lambda_{2}\left(2 \lambda_{1}+\beta\right) \\
+\left(\mathrm{p}_{1}-1\right) \lambda_{1} \beta
\end{array}\right]\right\} \exp \left\{\delta \lambda_{2}\right\} \\
-\lambda_{1}^{2} \lambda_{2}
\end{array} \lambda_{1} \lambda_{2}\left(\lambda_{1}+\beta\right)\left(\lambda_{2}+\beta\right)\right.}{\left.\lambda_{2}\right)}
\end{aligned}
$$

That is, cold duplication with imperfect switch of the system component increases the mean time to system failure by the amount:

$$
\left[\begin{array}{c}
\frac{\mathrm{p}_{1}\left[\left(2 \mathrm{p}_{1}-1\right) \lambda_{1}+\left(\mathrm{p}_{1}-1\right) \beta\right]}{\lambda_{1}\left(\lambda_{1}+\beta\right)}+\frac{\mathrm{p}_{2}^{2}\left(2 \lambda_{2}+\beta\right) \exp \left\{2 \delta \lambda_{2}\right\}}{\lambda_{2}\left(\lambda_{2}+\beta\right)} \\
+\frac{\mathrm{p}_{2}\left[\left(\begin{array}{l}
\left(\lambda_{1}+\lambda_{2}+\beta\right)\left(\begin{array}{l}
\mathrm{p}_{1} \lambda_{2}\left(2 \lambda_{1}+\beta\right)+ \\
\left(\mathrm{p}_{1}-1\right) \lambda_{1} \beta
\end{array}\right)-\lambda_{1}^{2} \lambda_{2}
\end{array}\right] \exp \left\{\delta \lambda_{2}\right\}\right.}{\lambda_{1} \lambda_{2}\left(\lambda_{1}+\beta\right)\left(\lambda_{2}+\beta\right)}
\end{array}\right]
$$

\section{MATERIALS AND METHODS}

The $\alpha$-fractiles: Let $L(\alpha)$ be the a-fractile of the original system and $\mathrm{L}^{\mathrm{D}}(\alpha), \mathrm{D}=\mathrm{H}, \mathrm{C}, \mathrm{I}$, are the $\alpha$ fractiles of the improved systems. The $\alpha$-fractiles $\mathrm{L}(\alpha)$ and $\mathrm{L}^{\mathrm{D}}(\alpha)$ are defined as the solution of the following equations, respectively:

$\mathrm{R}\left(\frac{\mathrm{L}(\alpha)}{\Lambda}\right)=\alpha, \mathrm{R}^{\mathrm{D}}\left(\frac{\mathrm{L}^{\mathrm{D}}(\alpha)}{\Lambda}\right)=\alpha$

Where: $\Lambda=\lambda_{1}+\lambda_{2}$

It follows from Eq. (2b) and the first Eq. (4a) that $\mathrm{L}=$ $\mathrm{L}(\alpha)$, satisfies the following equation:

$$
\mathrm{p}_{1} \exp \left\{-\frac{\lambda_{1} \mathrm{~L}}{\Lambda}\right\}+\mathrm{p}_{2} \exp \left\{-\lambda_{2}\left(\frac{\mathrm{L}}{\Lambda}-\delta\right)\right\}=\alpha
$$

From the second Eq. 4a, when $\mathrm{D}=\mathrm{H}$ and Eq. 3c, one can verify that $\mathrm{L}=\mathrm{L}^{\mathrm{H}}(\alpha)$ satisfies the following equation:

$$
\begin{aligned}
& 2 \mathrm{p}_{1} \exp \left\{-\frac{\lambda_{1} \mathrm{~L}}{\Lambda}\right\}+2 \mathrm{p}_{2} \exp \left\{-\lambda_{2}\left(\frac{\mathrm{L}}{\Lambda}-\delta\right)\right\} \\
& -\mathrm{p}_{1}^{2} \exp \left\{-\frac{2 \lambda_{1} \mathrm{~L}}{\Lambda}\right\}-\mathrm{p}_{2}^{2} \exp \left\{-2 \lambda_{2}\left(\frac{\mathrm{L}}{\Lambda}-\delta\right)\right\}- \\
& 2 \mathrm{p}_{1} \mathrm{p}_{2} \exp \left\{-\left(\mathrm{L}-\lambda_{2} \delta\right)\right\}=\alpha
\end{aligned}
$$

Similarly, from Eq. 3e and the second Eq. of 4a, when $\mathrm{D}=\mathrm{C}, \mathrm{L}=\mathrm{L}^{\mathrm{C}}(\alpha)$ can be obtained by solving the following equation:

$$
\begin{aligned}
\mathrm{p}_{1}^{2}\left(1+\lambda_{1} \frac{\mathrm{L}}{\Lambda}\right) \exp \left\{-\frac{\lambda_{1} \mathrm{~L}}{\Lambda}\right\}+\frac{2 \mathrm{p}_{1} \mathrm{p}_{2} \exp \left\{\lambda_{2} \delta\right\}}{\lambda_{2}-\lambda_{1}} \\
{\left[\lambda_{2} \exp \left\{-\frac{\lambda_{1} \mathrm{~L}}{\Lambda}\right\}-\lambda_{1} \exp \left\{-\frac{\lambda_{2} \mathrm{~L}}{\Lambda}\right\}\right] } \\
+\mathrm{p}_{2}^{2}\left(1+\frac{\lambda_{2} \mathrm{~L}}{\Lambda}\right) \exp \left\{-\lambda_{2}\left(\frac{\mathrm{L}}{\Lambda}-2 \delta\right)\right\}=\alpha
\end{aligned}
$$

Finally, from equation (3g) and the second equation of (4a), when $D=I, L=L^{\mathrm{I}}(\alpha)$ satisfies the following equation:

$$
\begin{aligned}
& \frac{\mathrm{p}_{1}^{2}}{\beta}\left(\lambda_{1}+\beta-\lambda_{1} \exp \left\{-\frac{\beta \mathrm{L}}{\Lambda}\right\}\right) \exp \left\{-\frac{\lambda_{1} \mathrm{~L}}{\Lambda}\right\} \\
& +\frac{\mathrm{p}_{1} \mathrm{p}_{2} \exp \left\{\lambda_{2} \delta\right\}}{\lambda_{1}+\beta-\lambda_{2}} \\
& {\left[\begin{array}{r}
\left.\left(\lambda_{1}+\beta\right) \exp \left\{-\frac{\lambda_{2} \mathrm{~L}}{\Lambda}\right\}-\lambda_{2}\right] \\
\left.\exp \left\{-\frac{\left(\lambda_{1}+\beta\right) \mathrm{L}}{\Lambda}\right\}\right]
\end{array}\right.} \\
& +\frac{\mathrm{p}_{1} \mathrm{p}_{2} \exp \left\{\lambda_{2} \delta\right\}}{\lambda_{2}+\beta-\lambda_{1}}\left[\left(\lambda_{2}+\beta\right) \exp \left\{-\frac{\lambda_{1} \mathrm{~L}}{\Lambda}\right\}-\lambda_{1}\right. \\
& \left.\exp \left\{-\frac{\left(\lambda_{2}+\beta\right) \mathrm{L}}{\Lambda}\right\}\right] \\
& +\frac{\mathrm{p}_{2}^{2} \exp \left\{2 \lambda_{2} \delta\right\}}{\beta}\left[\lambda_{2}+\beta-\lambda_{2} \exp \left\{-\frac{\beta \mathrm{L}}{\Lambda}\right\}\right] \\
& \exp \left\{-\frac{\left(\lambda_{2}+\mathrm{L}\right)}{\Lambda}\right\}=\alpha
\end{aligned}
$$


Equation 4b-4e has no closed form solutions and can be solved using some numerical program such as mathematic program system.

The survival reliability equivalence factor: $\mathrm{We}$ shall derive the SREF, when the mixing failure lifetime of the system component are reduced by the factor $\rho$, these factors will be denoted by $\rho^{\mathrm{D}}(\alpha)$, $D=H, C, I$. The factor $\rho^{D}(\alpha)$ is defined as the solution $\rho$ of the equation:

$\mathrm{R}^{\mathrm{D}}(\mathrm{t})=\mathrm{R}_{\rho}(\mathrm{t})=\alpha$

Using Eq. 3a together with Eq. 4f, one can verify that $\rho_{\mathrm{i}}=\rho_{\mathrm{i}}^{\mathrm{D}}$ satisfies the following system of equations:

$$
\left.\begin{array}{l}
\mathrm{p}_{1} \exp \left\{-\rho_{1} \lambda_{1} \mathrm{t}\right\}+\mathrm{p}_{2} \exp \left\{-\rho_{2} \lambda_{2}(\mathrm{t}-\delta)\right\}=\alpha \\
\mathrm{R}^{\mathrm{D}}(\mathrm{t})=\alpha
\end{array}\right\}
$$

By using Eq. 4g, together with Eq. 3c, 3e and 3g, one can verify that the factor $\rho_{\mathrm{i}}^{\mathrm{H}}, \rho_{\mathrm{i}}{ }^{\mathrm{C}}, \rho_{\mathrm{i}}{ }^{\mathrm{I}}, \mathrm{i}=1,2$, satisfies the systems of equations which have no closed form solutions and can be solved using some numerical program such as mathematic program system.

The mean reliability equivalence factor: The MREF, say $\zeta^{\mathrm{D}}$, for $\mathrm{D}=\mathrm{H}, \mathrm{C}$, I can be obtained by solving the following equation:

$$
\operatorname{MTTH} \rho=\operatorname{MTTF}^{\mathrm{D}}
$$

Using Eq. $3 b$ together with Eq. 3 h, one can verify that $\zeta_{\mathrm{i}}=\zeta_{\mathrm{i}}^{\mathrm{D}}, \mathrm{i}=1,2$ satisfies the equation:

$$
\frac{\mathrm{p}_{1}}{\lambda \xi_{1}}+\frac{\mathrm{p}_{2} \exp \left\{\lambda_{2} \xi_{2} \delta\right\}}{\lambda_{2} \xi_{2}}=\mathrm{MTTF}^{\mathrm{D}}
$$

Equation (4i) can be solved numerically by using Mathematic Program System, to get $\zeta_{\mathrm{i}}^{\mathrm{D}}$ for given $\lambda_{\mathrm{i}}, \delta$ and $\mathrm{MTTF}^{\mathrm{D}}$. The $\mathrm{MTTF}^{\mathrm{D}}$ are given, for $\mathrm{D}=\mathrm{H}, \mathrm{C}$ and $\mathrm{I}$, from Eq. 3d, 3f and $3 \mathrm{~h}$ respectively.

\section{RESULTS}

To explain how one can utilize the previously obtained theoretical results, we introduce a numerical example. In such example, we calculate the two different reliability equivalence factors of a system of one component with two non-identical mixing lifetimes, under the following assumptions:

- The failure rates of the mixing lifetime is $\lambda_{1}=0.07$, $\lambda_{2}=0.09$

- $\quad$ The probability $\mathrm{p}=0.4$

- The system reliability will be improved when the system component of mixing lifetimes are improved according to one of the previous duplication methods

- In the reduction method, we improve the system reliability when the failure rates of mixing lifetime are reducing by the factor, $\rho_{\mathrm{i}}, \mathrm{i}=1,2$

- In the imperfect switch duplication method, $\beta=0.01$

- The delayed time $\delta=0.03$

For this example, we have found that, the mean time to failure of the original and improved systems assuming HDM, CDM, IDM are presented in Table 1. The $\alpha$-fractiles $\mathrm{L}(\alpha), \mathrm{L}^{\mathrm{D}}(\alpha)$ and the reliability equivalence factors $\rho^{\mathrm{D}}(\alpha), \mathrm{D}=\mathrm{H}, \mathrm{C}, \mathrm{I}$ are calculated using Mathematica Program System according to the previous theoretical formulae. In such calculations the level $\alpha$ is chosen to be $0.1,0.2, \ldots, 0.9$.

Table 2 represents the $\alpha$-fractiles of the original and improved systems that are obtained by improving the system component according to the previously mentioned methods. Table 3 shows the SREF of the improved systems using each duplication method. Table 4 shows the MREF of the improved systems using each duplication method.

Table 1: The MTTF of the original and improved systems
\begin{tabular}{llll}
\hline MTTF & MTTF $^{\mathrm{H}}$ & MTTF $^{\mathrm{I}}$ & MTTF $^{\mathrm{C}}$ \\
\hline 12.3990 & 18.6362 & 23.4532 & 24.8382 \\
\hline
\end{tabular}

Table 2: The $\alpha$-fractiles of the original and improved system

\begin{tabular}{lllll}
\hline$\alpha$ & $\mathrm{L}$ & $\mathrm{L}^{\mathrm{H}}$ & $\mathrm{L}^{\mathrm{I}}$ & $\mathrm{L}^{\mathrm{C}}$ \\
\hline 0.1 & 4.5737 & 5.9289 & 7.3153 & 7.7472 \\
0.2 & 3.1808 & 4.4642 & 5.6070 & 5.9384 \\
0.3 & 2.3729 & 3.5861 & 4.5564 & 4.8257 \\
0.4 & 1.8028 & 2.9420 & 3.7715 & 3.9945 \\
0.5 & 1.3623 & 2.4206 & 3.1267 & 3.3116 \\
0.6 & 1.0035 & 1.9702 & 2.5628 & 2.7144 \\
0.7 & 0.7008 & 1.5601 & 2.0436 & 2.1645 \\
0.8 & 0.4392 & 1.1646 & 1.5378 & 1.6288 \\
0.9 & 0.2089 & 0.7468 & 0.9992 & 1.0584 \\
\hline
\end{tabular}


J. Math. \& Stat., 7 (3): 169-176, 2011

Table 3: The SREF $\rho^{\mathrm{D}}(\alpha)$

\begin{tabular}{|c|c|c|c|c|c|c|c|c|c|}
\hline \multirow[b]{2}{*}{$\alpha$} & \multicolumn{3}{|c|}{$\rho_{2}{ }^{\mathrm{D}}=1$} & \multicolumn{3}{|c|}{$\rho_{1}{ }^{D}=1$} & \multicolumn{2}{|c|}{$\rho_{1}{ }^{\mathrm{D}}=\rho_{2}{ }^{\mathrm{D}}=1$} & \multirow[b]{2}{*}{$\rho^{c}$} \\
\hline & $\rho_{1}{ }^{\mathrm{H}}$ & $\rho_{1}{ }^{\mathrm{I}}$ & $\rho_{1}{ }^{\mathrm{C}}$ & $\rho_{2}{ }^{\mathrm{H}}$ & $\rho_{2}{ }^{I}$ & $\rho_{2}{ }^{\mathrm{C}}$ & $\rho^{\mathrm{H}}$ & $\rho^{I}$ & \\
\hline 0.1 & 0.6274 & 0.4655 & 0.4327 & 0.6443 & 0.4789 & 0.4447 & 0.7713 & 0.6251 & 0.5902 \\
\hline 0.2 & 0.4982 & 0.3386 & 0.3102 & 0.5710 & 0.4085 & 0.3774 & 0.7123 & 0.5671 & 0.5354 \\
\hline 0.3 & 0.3811 & 0.2286 & 0.2038 & 0.5056 & 0.3490 & 0.3205 & 0.6614 & 0.5205 & 0.4914 \\
\hline 0.4 & 0.2633 & 0.1205 & 0.0991 & 0.4409 & 0.2920 & 0.2659 & 0.6124 & 0.4776 & 0.4509 \\
\hline 0.5 & 0.1374 & 0.0067 & NA & 0.3733 & 0.2335 & 0.2099 & 0.5623 & 0.4352 & 0.4108 \\
\hline 0.6 & NA & NA & NA & 0.2991 & 0.1702 & 0.1491 & 0.5085 & 0.3908 & 0.3689 \\
\hline 0.7 & NA & NA & NA & 0.2133 & 0.0977 & 0.0794 & 0.4481 & 0.3419 & 0.3228 \\
\hline 0.8 & NA & NA & NA & 0.1066 & 0.0079 & 0.0070 & 0.3755 & 0.2842 & 0.2683 \\
\hline 0.9 & NA & NA & NA & 0.0454 & NA & NA & 0.2766 & 0.2065 & 0.1949 \\
\hline
\end{tabular}

\begin{tabular}{lll} 
Table 4: The MREF, $\zeta^{\mathrm{D}}$ & \\
\hline$\zeta_{2}{ }^{\mathrm{D}}=1$ & $\zeta_{1}{ }^{\mathrm{D}}=1$ & $\zeta_{1}{ }^{\mathrm{D}}=\zeta_{2}{ }^{\mathrm{D}}$ \\
\hline$\zeta_{1}{ }^{\mathrm{H}}=0.4781$ & $\zeta_{2}{ }^{\mathrm{H}}=0.5166$ & $\zeta^{\mathrm{H}}=0.6649$ \\
$\zeta_{1}{ }^{\mathrm{I}}=0.3408$ & $\zeta_{2}{ }^{\mathrm{I}}=0.3762$ & $\zeta^{\mathrm{I}}=0.5283$ \\
$\zeta_{1}{ }^{\mathrm{C}}=0.3148$ & $\zeta_{2}{ }^{\mathrm{C}}=0.3489$ & $\zeta^{\mathrm{C}}=0.4988$ \\
\hline
\end{tabular}

\section{DISCUSSION}

From Table 1, one can conclude that:

$\mathrm{MTTF}<\mathrm{MTTF}^{\mathrm{H}}<\mathrm{MTTF}^{\mathrm{I}}<\mathrm{MTTF}^{\mathrm{C}}$

Based on the results presented in Table 2, it seems that: $\mathrm{L}(\alpha)<\mathrm{L}^{\mathrm{H}}(\alpha)<\mathrm{L}^{\mathrm{I}}(\mathrm{a})<\mathrm{L}^{\mathrm{C}}(\alpha)$ in allstudied cases.

This is confirmed by the results obtained for MTTF. According to the results presented in Table 3, it may be observed that:

Hot duplication of the mixing lifetime will increase $\mathrm{L}$ (0.1) from $\frac{4.5737}{\Lambda}$ to $\frac{5.9289}{\Lambda}$ Table 2. The same effect on $\mathrm{L}(0.1)$ can occur by reducing the failure rates of (i) the first type of mixing lifetimes by the factor $\rho_{1}{ }^{\mathrm{H}}=$ 0.6274 (ii) the second type of mixing lifetimes by the factor $\rho_{2}{ }^{\mathrm{H}}=0.6442$, (iii) the two types of mixing lifetimes by the factor $\rho^{\mathrm{H}}=0.7713$, Table 3 .

Imperfect duplication of the mixing lifetimes will increase $L(0.1)$ from $\frac{4.5737}{\Lambda}$ to $\frac{7.3153}{\Lambda}$ see Table 2. The same effect on $\mathrm{L}(0.1)$ can occur by reducing the failure rates of (i) the first type of mixing lifetimes by the factor $\rho_{1}{ }^{I}=0.4655$ (ii) the second type of mixing lifetimes by the factor $\rho_{2}{ }^{\mathrm{I}}=0.47842$, (iii) the two types of mixing lifetimes by the factor $\rho^{\mathrm{I}},=0.6251$, see Table 3

Cold duplication of the mixing lifetime will increase $\mathrm{L}(0.1)$ from $\frac{4.5737}{\Lambda}$ to $\frac{7.7472}{\Lambda}$ Table 2. The same effect on $\mathrm{L}(0.1)$ can occur by reducing the failure rates of (i) the first type of mixing lifetimes by the factor $\rho_{1}{ }^{\mathrm{C}}=$
0.4327 , (ii) the second type of mixing lifetimes by the factor $\rho_{2}{ }^{\mathrm{C}}=0.4447$, (iii) the two types of mixing lifetimes by the factor $\rho^{\mathrm{C}}=0.5902$, see Table 3 ,

In the same manner, one can read the rest of results presented in Tables 3 .

The notation NA, means that there is no equivalence between the two improved systems: one obtained by reducing the failure rates of the only one type of the mixing lifetime and the other obtained by improving the system component according to the duplication methods.

Based on the results presented in Table 4, one can conclude that.

The improved system that can be obtained by improving the mixing lifetime according to hot duplication method, has the same mean time to failure of that system which can be obtained by reducing (i) the first type of the mixing failure rates by the factor $\zeta_{1}{ }^{\mathrm{H}}=0.4781$, (ii) the second type of the mixing failure rates by the factor $\zeta_{2}{ }^{\mathrm{H}}=0.5166$, (iii) the two types of the mixing failure rates by the factor $\zeta^{\mathrm{H}}=0.6649$, Table 4 .

The improved system that can be obtained by improving the mixing lifetime according to imperfect duplication method, has the same mean time to failure of that system which can be obtained by reducing (i) the first type of the mixing failure rates by the factor $\zeta_{1}{ }^{\mathrm{I}}=0.3408$, (ii) the second type of the mixing failure rates by the factor $\zeta_{2}{ }^{\mathrm{I}}=0.3762$, (iii) the two types of the mixing failure rates by the factor $\zeta^{\mathrm{I}}=0.5283$, Table 4 .

The improved system that can be obtained by improving mixing lifetime according to cold duplication method, has the same mean time to failure of that system which can be obtained by reducing (i) the first type of the mixing failure rates by the factor $\zeta_{1}{ }^{\mathrm{C}}=0.3148$, (ii) the second type of the 
mixing failure rates by the factor $\zeta_{2}{ }^{\mathrm{C}}=0.3489$, (iii) the two types of the mixing failure rates by the factor $\zeta^{\mathrm{C}}=0.4988$, Table 4 .

\section{CONCLUSION}

The quality of the system reliability can be improved using four different methods of the system improvements. The results can be used to distinguish between the original and improved systems performances and calculate the equivalent between different cases of improving methods.

\section{REFERENCES}

Billinton, R. and R.R. Allan, 1983. Reliability Evaluation of Engineering Systems: Concepts and Techniques. Plenum 2nd Edn. Plenum Press, New York and London, ISBN: 978-0-306-44063-2, pp: 448.

El-Said, K.M. and M.S. El-Sherbeny, 2005. Evaluation of reliability and availability characteristics of two different systems by using linear first order differential equations. J. Math. Stat., 1:119-123. DOI: $10.3844 /$ jmssp.2005.119.123

Everitt, B.S. and D.J. Hand, 1981. Finite Mixture Distribution. 1st Edn., London New York, ISBN: 0412224208, pp: 143.

Gupta, R., S.K. Mittal and C.M. Batra, 2006. Stochastic analysis of a compound redundant system involving human failure. J. Math. Stat., 2: 407-413. DOI: $10.3844 /$ jmssp.2006.407.413

Haggag, M.Y., 2009. Cost analysis of two-dissimilarunit cold standby system with three states and preventive maintenance using linear first order differential equations. J. Math. Stat., 5: 395-400. DOI: $10.3844 / j m s s p .2009 .395 .400$

Lewis, E.E., 1996. Introduction to Reliability Engineering. 2nd Edn., John Wiley and Sons, Inc., New York, ISBN: 0471018333, pp: 435.

Mokaddis, G.S., M.S. El-Sherbeny and E. Al-Esayeh, 2009. Stochastics behavior of redundant complex system with two types of failure. J. Math. Stat., 5: 112-117. DOI: 10.3844/jmssp.2009.112.117

Mustafa, A. and A. El-Bassoiuny, 2009. Reliability equivalence of some systems with mixture linear increasing failure rates. Pak. J. Statis., 25: 149-163. ISSN: 1012-9367
Mustafa, A. and A.A. El-Faheem, 2011. Reliability equivalence factors of a system with $\mathrm{m}$ nonidentical mixed of lifetimes. Am. J. Applied Sci., $\quad 8$ : 297-302. 10.3844/ajassp.2011.297.302

Mustafa, A., 2008. Reliability equivalence of two nonidentical components. Int. J. Reliability Appl., 9: 79-93. ISSN: 1598-0073

Mustafa, A., 2009a. Reliability equivalence factor of ncomponents series system with non-constant failure rates. Int. J. Reliabil. Appl., 10: 43-45. ISSN: 1598-0073

Mustafa, A., 2009b, Reliability equivalence of some systems with mixture Weibull failure rates. African J. Math. Comput. Sci. Res., 2: 6-13. ISSN: 20069731

Mustafa, A., A. Sarhan and A.S. Al-Ruzaiza, 2007. Reliability equivalence of a parallel-series system. Pakist. J. Stati., 23: 241-254. ISSN: 1012-9367

Mustafa, A., B.S. El-Desouky and M. El-Dawoody, 2009. Reliability equivalence factors of nonidentical components series system with mixture failure rates. Int. J. Reliabil. Appl., 10: 17-32. ISSN: 1598-0073

Rushdi, A.M. and A.E. Alsulami, 2007. Cost Elasticities of Reliability and MTTF for k-out-of-n system. J. Math. Stat., 3: 122-128. DOI: 10.3844/jmssp.2007.122.128

Sarhan A. and A. Mustafa, 2006. Reliability equivalences of a series system consists of $n$ independent and non-identical components. Int. J. Reliabil. Appl., 7: 111-125. ISSN: 1598-0073

Sarhan, A.M., 2000. Reliability equivalence of independent and non-identical components series systems. Reliabil. Eng. Syst. Safet., 67: 293-300. DOI: 10.1016/S0951-8320(99)00069-1

Sarhan, A.M., 2002. Reliability equivalence with a basic series/parallel system. Applied Math. Comput., 132: 115-133. DOI: 10.1016/S00963003(01)00181-3

Sarhan, M., Tadj, L., Al-Khedhairi, A. and Mustafa, A. 2008. Equivalence factors of a parallel-series system. Applied Sci., 10: 219-230. ISSN: 14545101

Teamah, A.M. and A.M. El-Bar, 2009. Random sum of mixtures of sum of ivariate exponential distributions. J. Math. Statis., 5: 270-275. DOI: $10.3844 / j m s s p .2009 .270 .275$ 
Xia, Y. and G. Zhang, 2007. Reliability equivalence factors in gamma distribution. Applied Math. Comput., 187: 567-573. DOI: 10.1016/S00963003(01)00181-3 